\title{
Lung cancer screening in Europe: where are we in 2021?
}

\author{
Jan P. van Meerbeeck ${ }^{1,2}$, Caro Franck ${ }^{3}$ \\ ${ }^{1}$ Department of Pulmonology \& Thoracic Oncology, Antwerp University Hospital, Edegem, Belgium; ${ }^{2}$ Antwerp University, Antwerp, Belgium; \\ ${ }^{3}$ Department of Medical Imaging, Antwerp University Hospital, Edegem, Belgium \\ Contributions: (I) Conception and design: JP van Meerbeeck; (II) Administrative support: None; (III) Provision of study materials or patients: None; \\ (IV) Collection and assembly of data: None; (V) Data analysis and interpretation: None; (VI) Manuscript writing: All authors; (VII) Final approval of \\ manuscript: All authors. \\ Correspondence to: Jan P. van Meerbeeck. Department of Pulmonology \& Thoracic Oncology, Antwerp University Hospital, Edegem, Belgium. \\ Email: jan.van.meerbeeck@uza.be.
}

\begin{abstract}
This manuscript reviews the recent evidence obtained in lung cancer screening with low dose spiral CT-scan (LDSCT) and focuses on the issues associated with its implementation in Europe. After a review of the magnitude of the lung cancer toll in lives, disease and Euro's, the recently released data of the major lung cancer screening trials are reviewed and mirrored with the results of the US National Lung Screening Trial (NLST), comparing their strengths and weaknesses and areas of future research. The specific barriers and hurdles to be addressed for widely implementing this population screening in European countries are discussed, with special emphasis on the issues of inclusion of smokers, smoking cessation interventions, radiation injury and capacity planning. The pros and cons of including current smokers will be addressed together with the issue which is the better smoking cessation intervention. A medical physicist's view on radiation exposure and quality control will address concerns about radiation induced cancers. The downstream effects of a LDSCT screening program on the capacity of CT-scans, radiologists, thoracic surgeons and radiation oncologists will follow. An estimated roadmap for the future is sketched with the expected role of all key stakeholders. This roadmap reflects the opinion leader's reflections as expressed in a number of discussions with European health authorities, taking place as part of the recently released European Beating Cancer plan.
\end{abstract}

Keywords: Lung cancer; screening; stage I; CT-scan; radiation risk

Submitted Jul 27, 2020. Accepted for publication Feb 19, 2021.

doi: $10.21037 /$ tlcr-20-890

View this article at: http://dx.doi.org/10.21037/tlcr-20-890

\section{Lung cancer in Europe: the magnitude of the problem}

In the Global Burden of Disease Studies, lung cancer is a leading cause of years of life lost (YLL) and of disabilityadjusted life-years (DALYs): according to the Causes of Death Collaborators, it moved from the $17^{\text {th }}$ most prevalent cause of death in 1990 to the $14^{\text {th }}$ in 2007 and the $12^{\text {th }}$ in 2017 , representing a worldwide number of lung cancer deaths in 2017 of $1,883,100$ and a more than $29 \%$ increase compared to $2007(1,2)$. It furthermore ranks $17^{\text {th }}$ among the causes of DALY, representing $1.8 \%$ of DALYs in all ages and $3.9 \%$ of DALYs among adults aged $50-74$ years (3).

With more than 2,000,000 incidental cases and 1,760,000 deaths, lung cancer represents $11.6 \%$ of the 18.1 million new cases of cancer worldwide and $18.4 \%$ of its mortality (4). With a death rate of more than $85 \%$, lung cancer in Europe represents $3 \%$ of all causes of death and around $20 \%$ of cancer deaths, one third of them occurring in women. With one death every 83 seconds, the estimated number of European citizens dying from lung cancer in 2018 was 
$387,913$.

For Europe this incidence represents 470,039 new cases in 2018, i.e., 1 new case every minute (5). Incidence rates for males are highest in East-European countries like Hungary, Poland, Romania, Croatia and Latvia and for females in Denmark, Hungary, the Netherlands, Ireland and the United Kingdom. Lung cancer incidence in women has increased with $4.4 \%$ between 2014 and 2019, reaching a rate of $14.8 / 100,000$, whilst in men is declining but to a lesser extent than the increase in women, so that the net balance in Europe is an increase. The reader interested in country-specific incidence data is referred to the database of the International Agency for Cancer Research (6).

Besides this high incidence and mortality, lung cancer in Europe has a poor prognosis with a 5-year survival rate of $13 \%(11.2 \%$ in males, $13.9 \%$ in females) with distinct gradients between Northern, Southern, Eastern and Central Europe (7). This bad prognosis is obviously linked to its late diagnosis, which is typically in the advanced stage 4 in $50-70 \%$ of incidental cases, compared to $15-25 \%$ in the early stage 1.

The financial cost of lung cancer is huge: LuengoFernandez et al. estimated the total cost in 2009 to mount to $18,779 \mathrm{k} €$, of which $23 \%$ due to health care costs, $53 \%$ due to mortality losses, 20\% to informal care costs and 4\% to morbidity losses (8). Within the health care costs, $68 \%$ is attributed to inpatient expenditures, $12 \%$ to medicine, $13 \%$ to outpatient, $6 \%$ to primary care and $1 \%$ to the management of adverse events. Compared to breast, colon and prostate cancer, lung cancer has the highest total cost and the second highest inpatient expenditures after colorectal cancer. There are however large variations within Europe, with Luxembourg, Germany and the Netherlands spending on average 3-4 times more Euro's per capita than Lithuania, Cyprus and Bulgaria. As mentioned, these figures are for 2009, and it is estimated that the direct cost per patient increased more than 50\% between 2006-2016 and that these figures do not include the costs of novel targeted and immunotherapies, which came into standard practice later on. Recent retrospective data from the Belgian Health Technology Assessment Institute KCE show a tripling of the expenses for systemic therapy in stage 4 lung cancer after the introduction of the checkpoint inhibitors (M Neyt, personal communication, 2020).

Regarding treatment costs, large variations in cost/quality adjusted life year (QALY) are present according to stage and treatment modality: the incremental cost-effectiveness ratio (ICER) of a lobectomy in early stage disease amounts to
$10,000 € /$ QALY, of chemo-radiotherapy in locally advanced disease to $16,000 € / \mathrm{QALY}$, and of first line chemotherapy in advanced disease to $20,000 € / \mathrm{QALY}$ (9). Targeted agents and immunotherapy raise the bar to 25,000 €/QALY and $100,000 € / \mathrm{QALY}$, respectively. Notable disparities in access to new drugs exist between Western and Eastern Europe and should be jointly addressed by health authorities and pharmaceutical companies (10).

It is felt by most experts that any major decrease in mortality with ensuing decrease in expenditures and increase in survival can only be obtained by a diagnosis in an earlier asymptomatic stage and that pumping Euro's in inpatient care and medication will only exponentially increase as the incidence and prevalence of the disease is expected to rise further. There is hence an urgent need for a cost-effective screening tool for early detection of lung cancer. Low dose spiral CT-scan (LD-SCT) is likely to fulfill these promises.

\section{Lung cancer screening in Europe: efforts and endeavours}

Last year has seen the publication of the results of several European randomized trials in lung cancer screening: the Dutch-Belgian NELSON, the German LUSI and the mature Italian MILD (11-13). Their major characteristics and endpoints are summarized in Table 1. In all 4 trials a significant reduction in lung cancer specific mortality is noted, although expressed as various risk rates. Only the US NLST observed a significant reduction in overall mortality (14). Other trials were not powered to show this endpoint, which is considered by some health authorities to be a prerequisite for further implementation. Survival data of the incidental lung cancer cases are also not available, as the trials were not sufficiently powered to compare this outcome between both interventions. Nevertheless, the observed inversion of stage distribution with consistently more stage 1 tumours being found in the Ct-screened participants, is a accepted sufficient surrogate for an improvement in survival, as earlier stages have been shown to largely outlive advanced stages lung cancer (18).

A recent systematic review with meta-analysis of published data included seven randomised trials, which corresponds to 84,558 participants (19). A significant relative reduction of lung cancer-specific mortality of $17 \%$ ( $R R=0.83,95 \%$ CI: $0.76-0.91)$ and a relative reduction of overall mortality of $4 \%$ ( $R R=0.96,95 \%$ CI: $0.92-1.00)$ was observed in the screening group compared with the control group. Another meta-analysis, this time based on eight trials with 
Table 1 Randomised trials using low dose CT-scan: major trial characteristics and endpoints

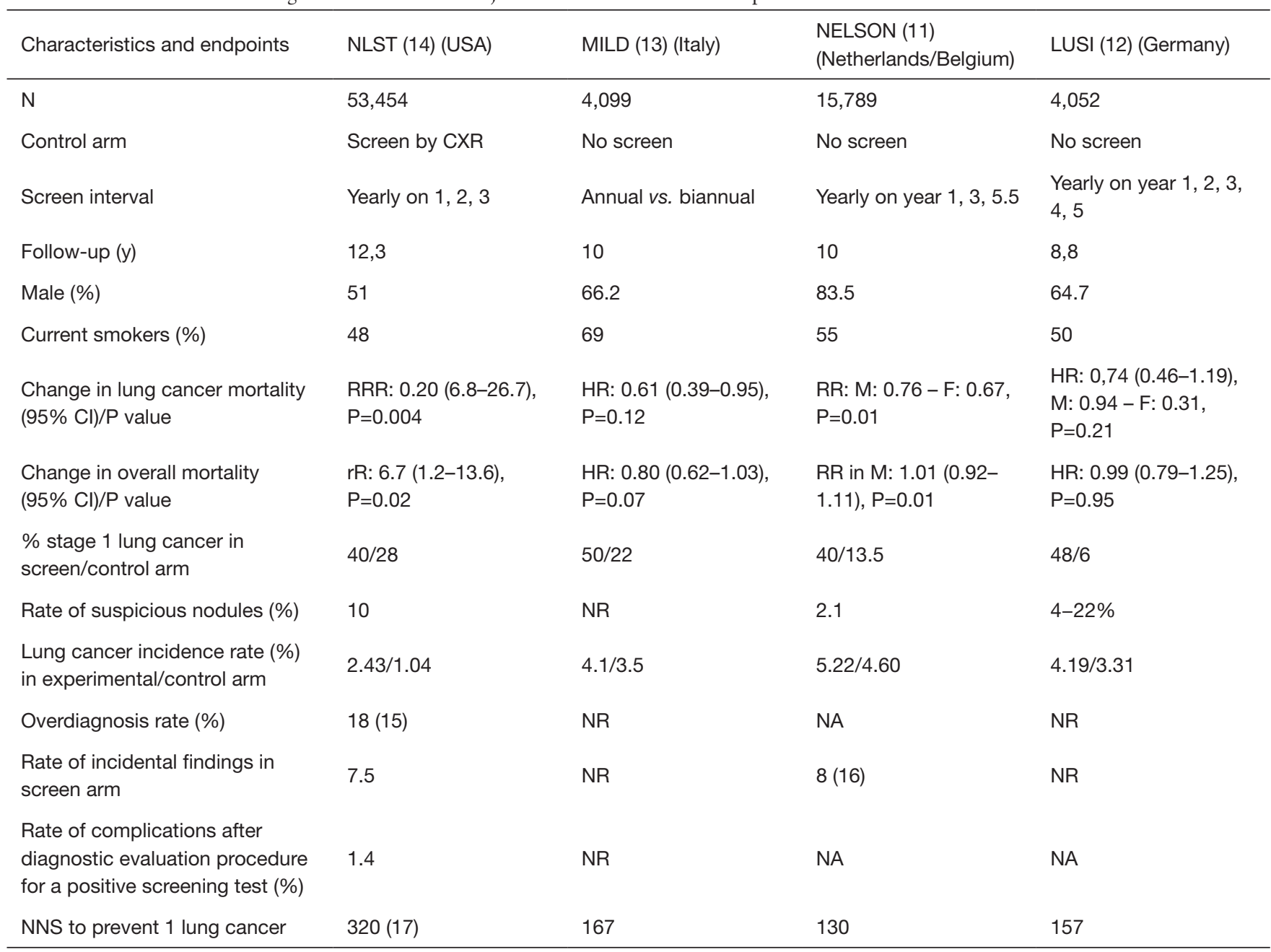

NLST, National Lung Screening Trial; MILD, Multicentric Italian Lung Detection; NELSON, NEderlands-Leuvens Longkanker Screening ONderzoek; LUSI, Lung cancer Screening Intervention; RRR, relative reduction rate; HR, hazard ratio; $r R$, reduction rate; RR, rate ratio; M, male; F, female; NNS, number needed to screen; $95 \% \mathrm{Cl}, 95 \%$ confidence interval; NA, not available; NR, not reported.

90,475 patients had a low risk of bias (20). There was a significant reduction in lung cancer-specific mortality with LD-SCT screening (relative risk $=0.81 ; 95 \% \mathrm{CI}$ : $0.74-0.89)$; the estimated absolute risk reduction was $0.4 \%$ (number needed to screen $=250$ ). The reduction in allcause mortality was not statistically significant (relative risk $=0.96$; $95 \%$ CI: $0.92-1.01$ ), but the absolute reduction in all-cause mortality was consistent with that for lung cancerspecific mortality $(0.34 \%$; number needed to screen $=294)$. In the studies with the longest duration of follow-up, the incidence of lung cancer was $25 \%$ higher in the screened group, corresponding to a $20 \%$ rate of overdiagnosis. Consensus hence grows that these results bring the necessary confirmation to the NLST and NELSON data and that further randomized trials addressing the issue of effectiveness of low dose CT-scan are futile and that the efforts should now direct at implementation in Europe.

Issues as false positive, overdiagnosis, lung cancer incidence and complication rates have variously been reported and do not all have standardized definitions, making inter-trial comparisons hazardous (15). The number needed to screen for 1 detected case varies from less than 300 in NLST to $\sim 130$ in NELSON. This reflects the importance of the nodule evaluation protocol, whereby the use of volumetric criteria and a $3^{\text {rd }}$ category of indeterminate nodules in NELSON has drastically reduced the number 
of so-called 'positive' scans reported in NLST (16). The role of artificial intelligence by deep machine learning is the focus of ongoing intensive research. Another secondary endpoint is the rate of incidental findings which seems to be quite similar in both largest trials at 7.5-8\%, with nearly all of them without important clinical relevance (21).

A weakness of the current evidence is the fact that the follow-up is based on cancer and hospital registry data and not on clinical follow-up. This makes it impossible to monitor issues like quality of life, patient-reported outcomes, and sustainability of smoking (cessation). Further implementation studies will therefore be needed.

A strength of NELSON is its population-driven design, whereby participants were recruited via a postal survey, minimizing bias compared to spontaneous self-application or selection via patient records of general practitioners. The role of the latter will nevertheless be crucial in further implementation.

Current cancer screening programmes identify the target population on the basis of age and/or sex. Lung cancer screening is likely to be the first largescale cancer screening programme that relies on additional risk factors to select the population at risk, mostly tobacco consumption. Both NELSON and NLST selected participants on the basis of age and smoking but other trials and pilot programmes have selected on the basis of multivariable risk prediction models. Lung cancer screening could hence become the first major targeted cancer screening programme (22). Opportunistic screening is available as a private service in some countries and is even covered by some regional insurance companies. The current status of LCS in individual European countries is reviewed in (23). At the time of writing, only the Croatian health authorities have started a national population-based screening for lung cancer with LD-SCT on October 1, 2020. German sickness funds are considering reimbursement as of 2022, but details of inclusion criteria and interval are lacking. Different pilot implementation studies are running or planned e.g., in Poland, UK, Germany, Italy, Norway, Spain. In other countries, lung cancer screening is actively discouraged by the authorities, as in France, where the health authorities hide behind a lack of strong evidence from a previous systematic review (24). The prospective phase 3 ' 4 -in the lung-run' trial will include 26,000 high risk participants from 6 European countries and randomize those with a normal baseline CT-scan between annual and risk-targeted biennial screening (25).

Several European scientific societies-among which the European Respiratory Society (ERS, the European Society of
Radiology (ESR), the European Society of Thoracic Imaging (ESTI), the European Society of Thoracic Surgeons (ESTS) have taken initiatives, together with an ad hoc collaboration among individual hospitals, European institutions and the medical imaging providers. Clearly, there is a need for a more structured approach to avoid redundancy, duplication and loss of resources. The European Commission has launched an ambitious 'Europe's Beating Cancer Plan' plan and has allocated funding to it (26). Unfortunately, the recent COVID-19 pandemic has halted-temporarily? - its course and it remains to be seen whether commissioner Stella Kyriakides, MEP, will be able to safeguard these funds in the ongoing financial and economic downfall of the pandemic.

\section{Lung cancer in Europe: challenges and strategies to surmount them}

Notwithstanding these efforts, the implementation of lung cancer screening in Europe will still meet formidable challenges. The latter can result in operational, psychological and financial barriers and have been either addressed in recent reviews and recommendations or are the subject of a number of manuscripts in this focused review (27-30) (Table 2). We will hence restrict here to the issues of inclusion of smokers, smoking cessation intervention, radiation injury and capacity planning.

\section{Smoking (cessation intervention)}

Opinions differ as to whether current smokers should be approached for lung cancer screening, as this would be perceived as a reward and approval of their habit. Others claim that lung cancer screening is an unique 'teachable moment' for the smoker to be approached and convinced for quitting. Few smokers are aware of CT-scan screening. Surveys among adult smokers and non-smokers investigated the acceptability and intention to participate in lung cancer screening. Their findings suggest a screening programme would be acceptable to the smoking and non-smoking population and also achieve high attendance rates (31-33). Utilization of lung cancer screening among smokers enlisted in a tobacco cessation programme is however low, as a large spectrum of barriers is present. Whether participation to a screening programme influences the quit rate is not clear: the existing evidence suggests that, by and large, this population is motivated to cease smoking (34). Smokingcessation interventions increased after new lung cancer screening guidelines. Given the sizable adverse impacts of 
Table 2 Challenges for implementing lung cancer screening with CT-scan
1. Minimize psychological, physical and radiation-associated harm
2. Cost-effectiveness
3. Recruitment and eligibility: optimal risk model
4. Participation and compliance in underserved high risk populations
5. Workforce and capacity: CT-scan, radiologists, thoracic surgeons, radiation apparatus, oncology nurses
6. Service implementation and quality assurance
7. Additional health interventions: e.g., smoking cessation, COPD and cardiac comorbidity
8. Incidental findings: emphysema, mediastinal tumours, coronary artery calcifications, interstitial lung fibrosis, etc.
9. Overdiagnosis and false positive rate
10.Lack of patient and clinician awareness

smoking on morbidity and mortality, small increases in the implementation of smoking-cessation interventions could have substantial public health benefits. Considering that lung cancer screening provides an opportunity to intervene with smoking in a high-risk population that might otherwise not seek or receive smoking cessation services, potentially reduced motivation to quit smoking among some lung cancer screening participants should not be viewed as a rationale to forgo smoking cessation services. Many smokers undergoing lung cancer screening are interested in quitting smoking and in receiving smoking-cessation treatment. Although screening alone appears to have little or no effect on smoking behavior, those who receive positive results may be especially likely to quit. The preliminary findings for lung cancer screening participants who receive smoking cessation interventions are promising. Insufficient research in this area should clearly not limit the provision of evidencebased smoking-cessation interventions for these smokers (35). Reported smoking cessation intervention studies are overall of poor to fair quality with significant potential for bias and limited generalizability (36). There is insufficient data to suggest a particular approach to smoking cessation counseling in the LDSCT screening setting. While no studies compared combined pharmacotherapy and counseling to counseling alone or compared the various pharmacologic agents, we identified several studies underway investigating new approaches. Multimodal, comprehensive, and evidencebased treatments yield better clinical outcomes for smokers than usual care (e.g., general advice to quit, provision of self-help materials). Moreover, because the motivation to quit smoking varies in this population, an opt-out tobaccotreatment approach may be preferred in which all smokers seeking lung cancer screening receive access to smokingcessation treatment regardless of their motivation. No studies have investigated universal cessation support or automatic referral to cessation support services for lung cancer screening services, but this is an important area that warrants further investigation. The optimal strategy for smoking cessation in patients undergoing LDSCT screening remains hence unclear. Future studies should focus on evaluating effectiveness and implementation of combined counseling and pharmacotherapy to optimize smoking cessation during LDCT screening. Eight clinical trials, seven funded by the National Cancer Institute and one by the Veterans Health Administration, address this gap and form the SCALE (Smoking Cessation within the Context of Lung Cancer Screening) collaboration (37).

\section{Radiation injury}

A screening program of an asymptomatic population should balance the potential benefits and the harmful effects to the individual and population as a whole. Lung cancer screening (LCS) can only be effective when its perks outweigh its risks or the potential hazards of the process. One of the harms in LDSCT is radiation-induced lung cancer, associated with CT examinations. Although the radiation dose to the lungs resulting from a single chest $\mathrm{CT}$ is low, the associated risk for the population is not negligible. The excess relative risk for lung cancer, which describes the hazard of tumor development due to radiation exposure, rises proportionally with the radiation dose used. The prospect is linked to the gender and smoking status of the participant, the age at the start of the screening programme, as well as to the number 
of screening rounds and possible other exposures due to follow-up imaging.

Conclusions from atomic bomb survivor studies show that the excess risk for radiation-induced cancer due to low dose exposures [less than 10 milliSievert $(\mathrm{mSv})$ ] is small, yet large uncertainties are associated with the risk models used (38). From the age of 50, which is often considered a starting point for LDSCT, radiation-induced cancer risks decrease significantly with increasing age of the participant (39). Alike breast cancer screening, a detection-over-induction ratio (DIR) can provide a good indication of the benefit-risk ratio of LDSCT. DIR represents the ratio of the number of screen detected tumors to the number of radiation-induced cancers. According to Law et al., screening is trivial for DIRvalues above 100, meaning 1 expected cancer induction per 100 screen detected tumors (40). This suggests that the risks are small compared with the years of life saved resulting from the screening programme. At all times, DIR must remain above the value of 10 which is considered the critical threshold for screening programmes.

In contrast to mammography screening, exposure from LDSCT is not limited to a single organ. Apart from the lungs, several other organs are included in or at the border of the scan field-of-view (FOV). When considering a LDSCT, as recommended by the ESTI $\left(\mathrm{CTDI}_{\mathrm{vol}}=1.6\right.$ milliGray (mGy), DLP $=65 \mathrm{mGycm}$ ) (41), the highest estimated organ doses include: female breasts (2.9 mGy), lungs (2.8 mGy), esophagus (2.7), liver (1.7 mGy), stomach (1.4 mGy) and thyroid (2.7 mGy). For a 50-year-old female participant, for which risks are the highest, this results in a lifetime attributable risk (LAR) of lung cancer incidence of $0.01 \%$. The relationship between smoking and radiation exposure is known in literature and has been described as at least additive, suggesting that the radiation risk from LDSCT screening could be higher in smokers than BEIR VII models predict (42). Due to differences in radiosensitivity, LAR of cancer incidence in the other organs in the FOV are at least an order of magnitude lower and unlikely to play any role in risk-benefit analyses (43). Averaged over four screening rounds, the results of the NELSON demonstrated a rate of screen detected lung cancers of $0.9 \%$. In combination with the demonstrated incidence rate, this leads to a DIR of 90 $(0.9 \% / 0.01 \%)$. One cannot consider the topic of lung cancer screening without discussing the definition of 'low dose CT-scan'. Practice parameters for lung cancer screening, published by the American College of Radiology and, more recently, the ESTI's guidelines on recommended CT-scan radiation dose levels are a first step towards standardization of low dose in LDSCT (44). Clinical implementation of these scan protocols should be performed by a medical physicist, in close collaboration with a chest radiologist. Intensive ongoing dose monitoring and the use of Diagnostic Reference Levels (DRLs) are crucial in a continual optimization process.

\section{Capacity planning}

An important downstream implication of lung cancer screening is its impact on workforce capacity of radiologists and surgeons in the management of screen-detected cases. A key capacity consideration is delivery of the necessary additional mentored training of radiologists to provide the requisite expertise for reporting low dose CT-scans and performing percutaneous lung biopsies (45). Delivering an effective high quality service will require investment in new equipment and staffing, with robust strategies to maximize output from existing resources. Numbers of CTscanners per million inhabitants vary between European countries from 8.9 in Hungary to 44.3 in Iceland (46). This will become an important operational bottleneck for implementation in countries with a low distribution of CTscanners.

Not unexpectedly, the increase of cases in earlier stage will give rise to more surgical procedures. Blom et al. have modeled the treatment capacity required for the implementation of lung cancer screening in the USA using different simulations (47). They estimated that full-scale implementation of lung cancer screening causes a major increase in surgical demand, with a peak within the first 5 years: $37 \%$ more lung cancer surgeries in 2015-2040 than no screening, $2.2 \%$ less radiotherapy, and 5.4\% less chemotherapy. Adherence to screening strongly influenced results. A gradual buildup of adherence over different years can spread this peak over time. Careful surgical capacity planning is essential for successfully implementing screening, as the case load for the thoracic surgery workforce will increase and is incumbent upon the current workforce to continually improve outcomes in this patient population (48). Similar data are not yet available for Europe, although the European Society of Thoracic Surgeons (ESTS) has issued recommendations regarding the training and clinical profile for surgeons participating in screening programs (49).

An important yet unaccounted consideration is the impact of body radiation therapy for the primary treatment of early stage lung cancer for high-risk surgical patients (50). The outcomes of stereotactic body radiation therapy seem 
to be promising, and its impact on operable lung cancer cases and on workforce planning remains to be seen and will become an essential consideration. In the absence of randomized controlled trials showing equivalence of this radiation therapy modality to surgery, it could be argued that the proportion of patients actually undergoing a nonoperative form of therapy will not change.

\section{Lung cancer in Europe: roadmap and future perspectives}

The implementation of lung cancer screening in Europe will be a long and winding road: it took the USA as a single country 3 years between the publication of the NLST and the recommendation of the US Preventive services Task force (51). The implementation guide of the American Thoracic society and the American Lung Association was first published in 2018 (52). Despite this head start, adherence to the program in the USA is reportedly low: in 2016, $7.3 \%$ of eligible smokers participated, compared to $70 \%$ for breast cancer screening (53). These figures are sobering, as an adherence rate of at least $40 \%$ is required for a screening programme to be cost effective. Besides that, the fragmentation of health care systems in Europe will be a serious barrier for a rapid rolling-out of a uniform programme.

We foresee 4 stages before full implementation will be reached in Europe:

(I) In a first stage, national expert groups should be formed for the implementation of nationwide, population-based lung cancer screening. This experts groups should consist of key opinion leaders of relevant national medical societies in collaboration with national patient organisations and stakeholders at the national level (government, parliament). Advocacy should go in hand with raising public awareness by media and other communication channels, strive for approval of a screening programme with low-dose CT scan and formulation of standard operating procedures as well as nation-specific standards for infrastructure, pathways and outcomes/quality assurance measures based on nation-specific healthcare systems. The deliverable of these expert groups are:

i. An analysis of the requirements and procedures to be fulfilled at the national level for approval by health authorities;

ii. A benefit-harm analysis, including overdiagnosis, psychosocial effects and cost-effectiveness;

iii. An estimation of the needs in infrastructure and human resources;

iv. A gap analysis of the 'as is' and 'to be' scenarios;

v. An estimation of the needs in resources for implementation and performance.

(II) In a second stage, initiated in parallel with the previous one, advocacy at the EU level is built by relevant European medical scientific societies and organisations and in collaboration with respective national societies, European patient organisations and other potential stakeholders (54). This effort should concentrate on the development of a recommendation or even a directive by the European Council asking for implementation of nationwide, population-based lung cancer screening programme in EU countries. Besides, formulation of minimum standards and harmonization of procedures across the EU are on the agenda.

(III) In a third stage, at the EU-level, the aforementioned national guidelines will leave to a resolution by the European Commission to make lung cancer screening compulsory in Europe, but leaving the national authorities a window of opportunity to comply. This resolution will have to be translated at the national level by the local parliament and health authorities, keeping several degrees of freedom about the time frame and participant selection, interval and duration. Implementation in Europe will hence be asymmetrical, depending of the budgetary constraints and the capacity of the local health authorities

(IV) Finally, in a fourth stage, harmonization of the program across the EU will appear, as the evidence accumulates. This is the time to build an international data registry for benchmarking and to introduce value-based principles of management (55).

There is an important role for the different stakeholders involved (56):

(I) Physicians have key roles in the setup of multidisciplinary task forces with experts from many other fields to promote LCS, ensure quality and provide continuing medical education, as well as optimal communication, with the participants. Pulmonologists have a crucial role in identifying people eligible for LCS, reaching out to family doctors, sharing the decision-making process and promoting tobacco cessation. They need to ensure 
that the eligible risk population understands the importance of LCS and is informed of its potential benefits, risks and harms. The role of radiologists in LCS is to ensure that LDCT is optimised with regard to high image quality, minimum dose and the most appropriate management of screendetected "positive" nodules and incidental findings. Strict algorithms defining the exact workflow and procedures triggered by positive screen results and incidental findings have to be implemented, which involves thoracic oncologists, thoracic surgeons, pathologists and others. Thoracic surgeons and radiation oncologist should train and certify their fellows in parenchyma-sparing treatment alternatives as sublobar resection by video-assisted or robotic-assisted resection, stereotactic ablative radiotherapy and introducing new techniques as radiofrequency ablation by bronchoscopic navigation. General physicians, although already overloaded with administrative duties and with a keen skepticism against cancer screening, will have to be convinced to raise the issue of screening among their patientsmokers and convey objective information about risks and benefits in those in whom the inclusion criteria do not apply, e.g., light and never-smokers. All physicians involved should work in close collaboration with psychologist and social medicine experts in tobaccocessation intervention, as this will be considered the litmus test for the authorities to support any screening programme.

(II) Patients and their advocacy groups are invaluable partners to raise awareness among the general public, politicians and general physicians. Ideally, this should be done at the national and the European level, using all modern tools of communication: surveys, internet, Facebook, testimonies in newspapers, television, YouTube, ... Their message should counteract the perception among the public and the authorities that lung cancer screening in smokers is unethical. They have to translate the benefit in easy to understand messages, e.g., that the cost of screening is comprised in the excise taxes. Smoking cessation programmes should include information and contacts for screening opportunities and both should be advertised, e.g., on the cigarette package, in hospital lobbies and websites and by regular air time in the media.
(III) Industry partners, mostly vendors of CT-scan apparatus and of artificial intelligence for computeraided diagnosis should endeavor the acquisition of integrated, high performance and low dose high quality images, which are subsequently analysed by software programmes with a high negative predictive value, reducing as much as possible the reading time by the radiologists. Mobile scanning units are preferable in rural areas. Finally, there will be a need for a highly reliable and privacyproof central repository of Ct-images that can be consulted at any time by accredited radiologists.

(IV) Scientists: with the evidence present, we do not need further randomized trials on the issue whether lung cancer screening is (cost-)effective. We need large scale implementation studies at the national and European level, to address open issues as optimal interval, optimal participant selection, optimal duration, integration of smoking cessation programmes and the role of biomarkers, either as supplementary inclusion criterion before the CTscan, or to help in decision-making once a noncalcified nodule is found (57).

In conclusion, Europe is at the dawn of a new era in cancer prevention: the available evidence for lung cancer screening is in accordance with the adapted WHOrequirements for effective screening (58). Efforts should now turn towards implementing it in a progressive but irreversible manner in all EU countries and salvage the life by relieving the burden of thousands. For the time being, lung cancer screening with LDSCT should be offered to a high risk population of current and ex-smokers, on a repetitive-yearly?-basis and be accompanied by a comprehensive smoking cessation intervention.

\section{Acknowledgments}

Funding: None.

\section{Footnotes}

Provenance and Peer Review: This article was commissioned by the Guest Editors (Paul Van Schil and Annemiek Snoeckx) for the series "Lung cancer screening" published in Translational Lung Cancer Research. The article has undergone external peer review.

Conflicts of Interest: Both authors have completed the 
ICMJE uniform disclosure form (available at http://dx.doi. org/10.21037/tlcr-20-890). The series "Lung cancer screening" was commissioned by the editorial office without any funding or sponsorship. The authors have no other conflicts of interest to declare.

Ethical Statement: The authors are accountable for all aspects of the work in ensuring that questions related to the accuracy or integrity of any part of the work are appropriately investigated and resolved.

Open Access Statement: This is an Open Access article distributed in accordance with the Creative Commons Attribution-NonCommercial-NoDerivs 4.0 International License (CC BY-NC-ND 4.0), which permits the noncommercial replication and distribution of the article with the strict proviso that no changes or edits are made and the original work is properly cited (including links to both the formal publication through the relevant DOI and the license). See: https://creativecommons.org/licenses/by-nc-nd/4.0/.

\section{References}

1. GBD 2017 Disease and Injury Incidence and Prevalence Collaborators. Global, regional, and national incidence, prevalence, and years lived with disability for 354 diseases and injuries for 195 countries and territories, 1990-2017: a systematic analysis for the Global Burden of Disease Study 2017. Lancet 2018;392:1789-858.

2. GBD 2017 Causes of Death Collaborators. Global, regional, and national age-sex-specific mortality for 282 causes of death in 195 countries and territories, 1980-2017: a systematic analysis for the Global Burden of Disease Study 2017. Lancet 2018;392:1736-88.

3. GBD 2019 Diseases and Injuries Collaborators. Global burden of 369 diseases and injuries in 204 countries and territories, 1990-2019: a systematic analysis for the Global Burden of Disease Study 2019. Lancet 2020;396:1204-22.

4. Bray F, Ferlay J, Soerjomataram I, et al. Globocan 2018 Global cancer statistics 2018: GLOBOCAN estimates of incidence and mortality worldwide for 36 cancers in 185 countries. CA Cancer J Clin 2018;68:394-424.

5. Available online: https://ecis.jrc.ec.europa.eu/ explorer.php?\$0-0\$1-All\$2-All\$4-1,2\$3-22\$6-0,85\$52008,2008\$7-7\$CEstByCountry\$X0_8-3\$X0_19AE28E\$X0_20-No\$CEstRelative\$X1_8-3\$X1_9AE28\$X1_19-AE28E\$CEstByCountryTable\$X2_19AE28E. Accessed 18 June 2020.
6. Available online: https://ci5.iarc.fr/CI5I-X/Pages/table3_ sel.aspx . Accessed on February 1, 2021

7. De Angelis R, Sant M, Coleman MP, et al. Eurocare 5. TLO 2014 Cancer survival in Europe 1999-2007 by country and age: results of EUROCARE--5-a populationbased study. Lancet Oncol 2014;15:23-34.

8. Luengo-Fernandez R, Leal J, Gray A, et al. Economic burden of cancer across the European Union: a populationbased cost analysis. Lancet Oncol 2013;14:1165-74.

9. Vergnenègre $\mathrm{A}$, Chouaid C. Review of economic analyses of treatment for non-small-cell lung cancer (NSCLC). Expert Rev Pharmacoecon Outcomes Res 2018;18:519-28.

10. Available online: https://www.europarl.europa.eu/RegData/ etudes/STUD/2020/642388/IPOL_STU(2020)642388_ EN.pdf . Accessed on February 1, 2021

11. de Koning HJ, van der Aalst CM, de Jong PA, et al. Reduced Lung-Cancer Mortality with Volume CT Screening in a Randomized Trial. New Engl J Med 2020;382:503-13.

12. Becker N, Motsch E, Trotter A, et al. Lung cancer mortality reduction by LDCT screening-Results from the randomized German LUSI trial. Int J Cancer 2020;146:1503-13.

13. Pastorino U, Silva M, Sestini S, et al. Prolonged lung cancer screening reduced 10 -year mortality in the MILD trial: new confirmation of lung cancer screening efficacy. Ann Oncol 2019;30:1162-9.

14. National Lung Screening Trial Research Team, Aberle DR, Adams AM, et al. Reduced lung-cancer mortality with low-dose computed tomographic screening. N Engl J Med 2011;365:395-409.

15. Patz EF, Pinsky P, Gatsonis C, et al. Overdiagnosis in lowdose computed tomography screening for lung cancer. JAMA Intern Med 2014;174:269-74.

16. van Klaveren RJ, Oudkerk M, Prokop M, et al. Management of lung nodules detected by volume CT scanning. N Engl J Med 2009;361:2221-9.

17. O'Connor GT, Hatabu H. Lung cancer screening, radiation, risks, benefits, and uncertainty. JAMA 2012;307:2434-5.

18. Goldstraw P, Chansky K, Crowley J, et al. The IASLC Lung Cancer Staging Project: Proposals for Revision of the TNM Stage Groupings in the Forthcoming (Eighth) Edition of the TNM Classification for Lung Cancer. J Thorac Oncol 2016;11:39-51.

19. Sadate A, Occean BV, Beregi JP, et al. Systematic review and meta-analysis on the impact of lung cancer screening by low-dose computed tomography. Eur J Cancer 
2020;134:107-14.

20. Ebell MH, Bentivegna M, Hulme C. Cancer-Specific Mortality, All-Cause Mortality, and Overdiagnosis in Lung Cancer Screening Trials: A Meta-Analysis. Ann Fam Med 2020;18:545-52.

21. van de Wiel JCM, Wang Y, Xu DM, et al. Neglectable benefit of searching for incidental findings in the DutchBelgian lung cancer screening trial (NELSON) using lowdose multidetector CT. Eur Radiol 2007;17:1474-82.

22. Baldwin DR, Callister MEJ. An update on CT screening for lung cancer: the first major targeted cancer screening programme. Br J Radiol 2020;93:20200636.

23. Kauczor HU, Baird AM, Blum TG, et al. ESR/ERS statement paper on lung cancer screening. Eur Radiol 2020;30:3277-94.

24. Coureau G, Salmi LR, Etard C et al. Low-dose computed tomography screening for lung cancer in populations highly exposed to tobacco: A systematic methodological appraisal of published randomised controlled trials. Eur J Cancer 2016;61:146-56.

25. Available online: https://www.i-dna.org/4-in-the-lung-run/ . Accessed 1 February 2021

26. Available online: https://ec.europa.eu/commission/ presscorner/detail/en/SPEECH_20_195. Accessed 1 February 2021

27. Kauczor HU, Baird AM, Blum TG, et al. European Society of Radiology (ESR) and the European Respiratory Society (ERS) statement paper on lung cancer screening. Eur Respir J 2020;5 5:1900506.

28. Veronesi G, Baldwin DR, Henschke CI, et al. Recommendations of the Initiative for European Lung Screening (IELS) Group for the Implementation of LowDose CT Screening in Europe. Cancers 2020;12:1672.

29. Oudkerk M, Devaraj A, Vliegenthart R et al. European position statement on lung cancer screening. Lancet Oncol 2017;18:e754-66.

30. Field JK, Oudkerk M, Pedersen JH, Duffy SW. Prospects for population screening and diagnosis of lung cancer. Lancet 2013;382:732-41.

31. Quaife SL, Marlow LAV, McEwen A, et al. Attitudes towards lung cancer screening in socioeconomically deprived and heavy smoking communities: informing screening communication. Health Expect 2017;20:563-73.

32. Cataldo JK. High-risk older smokers' perceptions, attitudes, and beliefs about lung cancer screening. Cancer Med 2016;5:753-9.

33. Kellen E, Gabriels S, Van Hal G, et al. Lung cancer screening: intention to participate and acceptability among
Belgian smokers. Eur J Cancer Prev 2020. [Epub ahead pf print]. doi: 10.1097/CEJ.0000000000000656.

34. Raz DJ, Wu G, Nelson RA, et al. Perceptions and Utilization of Lung Cancer Screening Among Smokers Enrolled in a Tobacco Cessation Program. Clin Lung Cancer 2019;20:e115-e122.

35. Fucito LM, Czabafy S, Hendricks PS, et al. Pairing Smoking-Cessation Services With Lung Cancer Screening: A Clinical Guideline From the Association for the Treatment of Tobacco Use and Dependence and the Society for Research on Nicotine and Tobacco. Cancer 2016;122:1150-9.

36. Iaccarino JM, Duran C, Slatore CG, et al. Combining smoking cessation interventions with LDCT lung cancer screening: A systematic review. Prev Med 2019;121:24-32.

37. Joseph AM, Rothman AJ, Almirall D, et al. Lung Cancer Screening and Smoking Cessation Clinical Trials. SCALE (Smoking Cessation within the Context of Lung Cancer Screening) Collaboration Am J Respir Crit Care Med 2018;197:172-82.

38. Council NR, et al. Radiation, Health Risks from Exposure to Low Levels of Ionizing Radiation: BEIR VII Phase 2. National Academies Press, 2006.

39. Thompson DE, Mabuchi K, Ron E, et al. Cancer incidence in atomic bomb survivors. Part II: Solid tumors, 1958-1987. Radiat Res 1994;137:S17-67.

40. Law J, Faulkner K, Young KC. Risk factors for induction of breast cancer by X-rays and their implications for breast screening. Br J Radiol 2007;80:261-6.

41. European Society of Thoracic Imaging. ESTI LCS technical standards, 2019.

42. Albert JM. Radiation risk from CT: implications for cancer screening. AJR Am J Roentgenol 2013;201:W81-7.

43. Brenner DJ. Radiation risks potentially associated with low-dose CT screening of adult smokers for lung cancer. Radiology 2004;231:440-5.

44. Kazerooni EA, Austin JHM, Black WC, et al. ACRSTR practice parameter for the performance and reporting of lung cancer screening thoracic computed tomography (CT): 2014 (Resolution 4). J Thorac Imaging 2014;29:310-6.

45. British Society of Thoracic Imaging and the Royal College of Radiologists. Considerations to ensure optimum rollout of targeted lung cancer screening over the next five years. Available online: https://www.rcr.ac.uk/sites/default/ files/final_pdf_considerations_to_ensure_optimum_rollout_of_targeted_lung_cancer_screening.pdf

46. Available online: https://stats.oecd.org 
47. Blom EF, ten Haaf K, Arenberg DA, et al. Treatment capacity required for full scale implementation of lung cancer Screening in the United States. Cancer 2019; 125:2039-48.

48. Dhanasopon AP, Kim AW. Lung cancer screening and its impact on surgical volume. Surg Clin N Am 2017;97:751-62.

49. Pedersen JH, Rzyman W, Veronesi G, et al. Recommendations from the European Society of Thoracic Surgeons (ESTS) regarding computed tomography screening for lung cancer in Europe. Eur J Cardiothorac Surg 2017;51:411-20.

50. Nanda RH, Liu Y, Gillespie TW, et al. Stereotactic body radiation therapy versus no treatment for early stage nonsmall cell lung cancer in medically inoperable elderly patients: a National Cancer Data Base analysis. Cancer 2015;121:4222-30.

51. Humphrey LL, Deffebach M, Pappas M, et al. Screening for Lung cancer with low dose computed tomography: a systematic review to update the US Preventive Services Task force Recommendation. Ann Intern Med 2013;159:411-20.

52. Powell CA, Regis S, Rizzo A, et al. American Thoracic Society and American Lung Association. Lung Cancer Screening Implementation Guide. 2018.

Cite this article as: van Meerbeeck JP, Franck C. Lung cancer screening in Europe: where are we in 2021? Transl Lung Cancer Res 2021;10(5):2407-2417. doi: 10.21037/tlcr-20-890
53. Li J, Chung S, Wei EK, et al. New recommendations and coverage of low-dose computed tomography for lung cancer screening: uptake has increased but is still low. BMC Health Services Research 2018;18:52.

54. Horgan D, on behalf of European Alliance for Personalized Medicine. Revolutionising Europe's healthcare systems for lung cancer patients: A call for action on improving prevention, early detection, treatment and monitoring with new technologies. 2021.

55. Couespel N, on behalf of European Cancer Organisation. Strengthening Europe in the fight against cancer, study for the committee on Environment, Public Health and Food Safety, Policy Department for Economic, Scientific and Quality of Life Policies, European Parliament, Luxembourg, 2020.

56. Oudkerk M, Liu S, Heuvelmans MA, et al. Lung cancer LDCT screening and mortality reduction - evidence, pitfalls and future perspectives. Nat Rev Clin Oncol 2021;18:135-51.

57. Seijo LM, Peled N, Ajona D, et al. Biomarkers in Lung Cancer Screening: Achievements, Promises, and Challenges. J Thorac Oncol 2019;14:343-57.

58. Dobrow MJ, Hagens V, Chafe R, et al. Consolidated principles for screening based on a systematic review and consensus process. CMAJ 2018;190:E422-9. 